\title{
Thermographic imaging as alternative method in allergy diagnosis
}

\section{A comparative study}

\author{
Tomasz $\operatorname{Rok}^{1} \cdot$ Eugeniusz Rokita $^{1,2} \cdot$ Grzegorz Tatoń $^{1} \cdot$ Tomasz Guzik $^{3} \cdot$ \\ Tomasz Śliwa ${ }^{3}$
}

Received: 13 October 2015/Accepted: 29 June 2016/Published online: 13 July 2016

(c) The Author(s) 2016. This article is published with open access at Springerlink.com

\begin{abstract}
Allergen sensitization is being diagnosed by commonly available methods in clinical practice-skin prick tests (SPTs) and specific immunoglobulin E test (sIgE). Recently, a new thermographic (TH) method for the assessment of SPT was developed, and it was demonstrated that the TH measurements of forearm temperature distribution during SPT, supported by a mathematical model, offer a new quantification method of allergen-induced skin reactions. The aim of this study is a comprehensive comparison of the TH method with SPT and SIgE techniques. The studies were performed for a group of 51 patients. The SPT and sIgE examinations were done in a routine way. For TH analyses, set of thermograms of both forearms were acquired after prick and analyzed with the use of developed software. All results were converted into categorized scale for comparison. The collected results indicate high correlation coefficients between methods equal to $0.76-0.99$. Sensitivity and accuracy of $\mathrm{TH}$ assessment in respect of both SPT and sIgE methods is at good level (0.72-0.93). Acceptable level of specificity $0.60-0.88$ was also achieved for most allergic responses. Excellent agreement between SPT and sIgE methods was observed which makes the TH assessment competitive. Due to higher precision and
\end{abstract}

Tomasz Rok

tomasz.rok@uj.edu.pl

1 Department of Biophysics, Chair of Physiology, Jagiellonian University Medical College, Św. Łazarza 16, 31-530 Cracow, Poland

2 Institute of Physics, Faculty of Physics, Astronomy and Applied Computer Science, Jagiellonian University, Cracow, Poland

3 Department of Internal and Rural Medicine, Jagiellonian University Medical College, Cracow, Poland sensitivity of digital infrared technology, possibility of making error in diagnosis is significantly reduced. Additional advantage of the TH method relies on an estimation of the skin reactivity which allows highlighting the hypersensitivity patients and automatic correction of the diagnosis.

Keywords Thermography · Mathematical model · Skin prick tests $\cdot$ Specific immunoglobulin E

\section{Introduction}

It is obvious that for patients suffering from allergic symptoms a correct and comprehensive identification of allergic sensitization is of fundamental importance. Skin prick test (SPT) is the most widely used diagnostic method in allergy assessment [1]. SPT is minimally invasive, inexpensive, and the results are immediately available. A limitation is the fact that the test has to be performed by trained professionals. The second commonly used method is a specific immunoglobulin E test (sIgE). Specific $\operatorname{IgE}$ test is quantitative, repeatable and accurate in vitro assay for the measurement of allergen-specific IgE in human serum or plasma [2]. Unfortunately, both methods possess some limitations. If used not carefully, they can lead to discrepant conclusions [3, 4]. As an example of misdiagnosing the cockroach sensitization was presented [5, 6]. Considerable disagreement between SPT and SIgE for diagnosing of allergic sensitization in young children was also reported $[3,7,8]$. On the other hand, many authors correlated results of both methods, and a good agreement was announced [3, 9-11].

Recently, a new thermographic (TH) method for the assessment of allergen-induced skin reactions was 
developed $[12,13]$. It was demonstrated that the thermographic measurements of forearm temperature distribution during SPT, supported by a mathematical model, offer a new quantification of allergen-induced skin reactions. The spatiotemporal analysis of thermographic images allows description of the immediate allergic process more precise. Contrary to the SPT which considers only the final effect of the allergic response, the proposed method distinctly discriminates the processes of mediator transport and its concentration changes. Such advantages as high sensitivity and spatiotemporal monitoring of allergic reaction make this method a valuable supplement of the already existing techniques.

The aim of this study is a comprehensive comparison of the TH method with SPT and SIgE techniques. Additional advantages with respect to routine methods will be also discussed.

\section{Methods}

The studies were performed for a group of 51 patients (31 females and 20 males) aged from 18 to 65 years in accordance with the guidelines of the ethics committee of the Jagiellonian University Medical College. The SPT studies were done using the commercial diagnostic allergen panel (Allergopharma, Reinbeck, Germany). According to the routine procedure, a negative control solution ( $9 \mathrm{mg} \mathrm{NaCl}, 4 \mathrm{mg}$ phenol, $563 \mathrm{mg}$ glycerol and water for injection to $3 \mathrm{~mL}$ ) and a positive control fluid (control solution with $1.7 \mathrm{mg}$ of histamine hydrochloride) were used in the studies. The following basic inhalant allergens were considered: mixed weeds (mugwort, ribwort and nettle), mixed trees 1 (grey alder, hazel, poplar), mixed trees 2 (common silver birch, beech and oak), mixed grasses (timothy, orchard grass and meadow grass), Dermatophagoides pteronyssinus, Dermatophagoides farinae, mildews (Alternaria tenuis), molds fungi (Cladosporium herbarum), cat dander, dog dander and cultivated rye. The full panel of allergens extracts was not used for all examined patients. A standard medical interview and the qualification of patient were performed during an earlier visit, and then, $15 \mathrm{~mL}$ of blood for the sIgE test was collected.

The procedure of thermographic measurement only slightly differed from standard procedure of SPT. Patients were allowed to adapt for $30 \mathrm{~min}$ in order to stabilize the temperature of the skin. Afterward, single thermogram of both forearms was acquired in order to check the temperature stabilization. The skin prick tests were performed on the palmar surface of the forearms (at least $5 \mathrm{~cm}$ above the wrist and $3 \mathrm{~cm}$ below the elbow). Patient's forearms were fixed on a special table perpendicularly to the infrared camera. A thermographic camera (VIGO, Warsaw, Poland) was placed $\sim 30 \mathrm{~cm}$ above the forearms. Next, the standard procedure of skin prick test was performed. Drops of allergen extracts were placed onto marked areas of the skin. Using a sterile lancet, small pricks through the drop were vertically made. Then, a series of thermograms of both forearms were acquired every $70 \mathrm{~s}$. The acquisition time was about $15 \mathrm{~min}$. After $15 \mathrm{~min}$, skin responses for all allergens and both controls were evaluated using planimetric measurement by the well-experienced technician.

The routine standard SPT evaluation method considers a positive response if the largest diameter of the wheal of each particular test is $\geq 3 \mathrm{~mm}$ [1]. The following scale was adopted in the studies: $(0)$ - the same wheal diameter size as for negative control-no reaction, (1) — very small induration, erythema present-weak reaction (mild), (2) $\leq 50 \%$ of wheal diameter size compared to histamine control-moderate sensitivity, (3)-the wheal diameter size (50-100) \% of histamine control—definitely positive and (4) - the wheal diameter size larger than for histamine control or pseudopodia present-strongly positive.

The specific IgE tests were performed with the ImmunoCAP $^{\circledR}$ system (Phadia, Uppsala, Sweden). The concentration of $\operatorname{sIgE}$ was converted into nominal scale (classes) according to following rules: $<0.35 \mathrm{IU} \mathrm{mL}^{-1}$ level 0 (negative), (0.36-0.69) $\mathrm{IU} \mathrm{mL}^{-1}$-level 1 (borderline levels), (0.7-3.49) IU $\mathrm{mL}^{-1}$-level 2 (slightly elevated), (3.50-17.4) IU mL ${ }^{-1}$-level 3 (moderately elevated), (17.5-49.9) IU $\mathrm{mL}^{-1}$-level 4 (high levels), (50-100) IU $\mathrm{mL}^{-1}$-level 5 (very high levels) and $>100 \mathrm{IU} \mathrm{mL}^{-1}$-level 6 (extremely high levels). Among studied patients, the concentrations of sIgE higher than $50 \mathrm{IU} \mathrm{mL} \mathrm{m}^{-1}$ were not observed. For this reason, above scale was limited to five-point scale.

Thermograms were evaluated using the software developed in our laboratories [12]. Briefly, the first step of the analysis relies on the determination of the temperature increase $(\Delta T)$ distributions. For this purpose, subtraction of the image acquired before examination from images recorded at different time after allergen introduction was performed. In some cases, a correction for the forearm movement was applied. Next, each forearm region heated as the effect of skin response for allergen or positive control was approximated by a circle. The temperature increase distributions after histamine (control) and allergen introduction as a function of time $(t)$ and distance from the prick point $(r)$ were used to determine the model parameters $\left(\Delta T_{\mathrm{H}}(r, t)\right.$ and $\Delta T_{\mathrm{A}}(r, t)$, respectively).

In the model, it was assumed that histamine (positive control) is introduced at the origin $\left(c_{\mathrm{H}}(0,0)=c_{\mathrm{OH}}\right)$, and it is transported (migrates) across the skin. As histamine migrates, it is additionally eliminated via first-order kinetics. Hence, the histamine transport is described by 2 parameters: histamine migration rate $(v)$ and the 
elimination rate $(\gamma)$. As histamine is moving across the skin, the vessel system reacts in response to the local histamine level $\left(c_{\mathrm{H}}(r, t)\right)$. The engorged vessels are, in turn, responsible for skin redness and an increase in skin temperature. We assume that due to the histamine-induced vasodilatation a supplementary heat source $\left(Q_{\mathrm{H}}\right)$ is activated. At point $r$, a supplementary heat source appears after time $t=r / v$. The increase in heating is constant in time and is linearly related to the maximal histamine level at point $r\left(Q_{\mathrm{H}}(r)=Q_{0} c_{\mathrm{H}}(r, r / v)=Q_{0} c_{0 \mathrm{H}} \exp (-\gamma r / v), Q_{0}\right.$-positive constant).

The temperature distribution of the skin is described by the Pennes bio-heat equation with additional approximations that the thermal diffusivity in the skin and metabolic heat generation rate may be neglected. Hence, the temperature increase distribution is described by first-order linear differential equation which may be easy solved analytically. The solution contains many parameters which may be pooled into 2 quantities. The first one $(\tau)$ is a combination of the skin density, the specific heat of the skin, the blood perfusion, the density of blood, the specific heat of blood and the heat transfer coefficient. The second quantity $\left(S_{\mathrm{H}}\right)$ contains additionally the blood and ambient temperatures and the heat generation rate due to histamine action $\left(Q_{\mathrm{H}}\right)$.

In the modeling of the allergen action, IgE-mediated hypersensitivity is assumed. The exposure to the allergen leads to cross-linking of the IgE molecules on skin mast cells, and the cell releases histamine directly generating allergic symptoms. In the model, allergen molecules are injected to the skin, but they do not move comparing to histamine motion. The allergen conversion, via a mast cell, into histamine produces the concentration of histamine $\left(c_{\mathrm{OA}}\right)$ at the origin after allergen injection. Under the above-described assumptions, the temperature distribution after allergen injection is described by similar equation as after control histamine introduction with the new value $\left(S_{\mathrm{A}}\right)$ of the $S_{\mathrm{H}}$ constant. More precisely, $Q_{\mathrm{H}}(r)$ is replaced by $Q_{\mathrm{A}}(r)=Q_{0} c_{0 \mathrm{~A}} \exp (-\gamma r / v)$. The histamine production after allergen injection, i.e., the allergic response $(\mathrm{TH}$ diagnostic parameter), may be quantified by the $c_{\mathrm{OA}}$ value or the $Q_{\mathrm{A}} / Q_{\mathrm{H}}=c_{\mathrm{OA}} / c_{\mathrm{OH}}$ ratio. The $\Delta T_{\mathrm{H}}(r, t)$ distribution was used to determine the model parameters, describing the increase in the skin temperature after histamine injection $\left(v, \gamma, \tau, Q_{\mathrm{H}}\right)$. The parameter characterizing the temperature distribution after allergen injection $\left(Q_{\mathrm{A}}\right)$ was determined by fitting the model equation to the $\Delta T_{\mathrm{A}}(r, t)$ data. It should be pointed out that for each patient the values of $v, \gamma, \tau, Q_{\mathrm{H}}$ were extracted from analysis of the histamine data. Therefore, the evaluation of the allergen data relied on the determination of the $Q_{\mathrm{A}}$ value.

Similarly to SPT and sIgE methods, the TH results were categorized. The following scale was adopted for the investigated allergens: (0)— the same value of diagnostic parameter as negative control, (1)-larger than negative but smaller than $50 \%$ of the histamine control-weak reaction (mild), (2)-(51-90\%) of histamine controlmoderate sensitivity, (3)-(91-130\%) of histamine control-definitely positive, (4) — the value of parameter larger than $130 \%$ of histamine control-strongly positive.

Commercially available Statistica 10 package (StatSoft, Poland) was used for statistical analysis. $95 \%$ confidence level was applied. Gamma correlation rank test was used for the assessment of correlations between methods. Statistical differences between methods and particular allergens were tested with nonparametric Kruskal-Wallis test. The Kolmogorov-Smirnov test was used in order to compare diameters of the skin wheals.

\section{Results}

Representative infrared thermogram of the right forearm (left image) and the resultant image after subtraction of the thermal image acquired before examination from the last thermal image recorded during SPTs (right image) are presented in Fig. 1. It should be noted that applied procedure of thermal image analysis significantly enhances the segmentation of skin lesions. Allergic skin reactions are more evident and therefore easier for analysis.

The maximum number of allergen extracts tested for a single patient was 11 , while minimum number was 8 . The sensitization was quantified using the above-described categorized scale. The mildews and molds fungi were excluded from further analysis due to too low number of positive results ( 4 cases). The total number of allergic tests, both positive and negative, among all of 51 patients was 387.
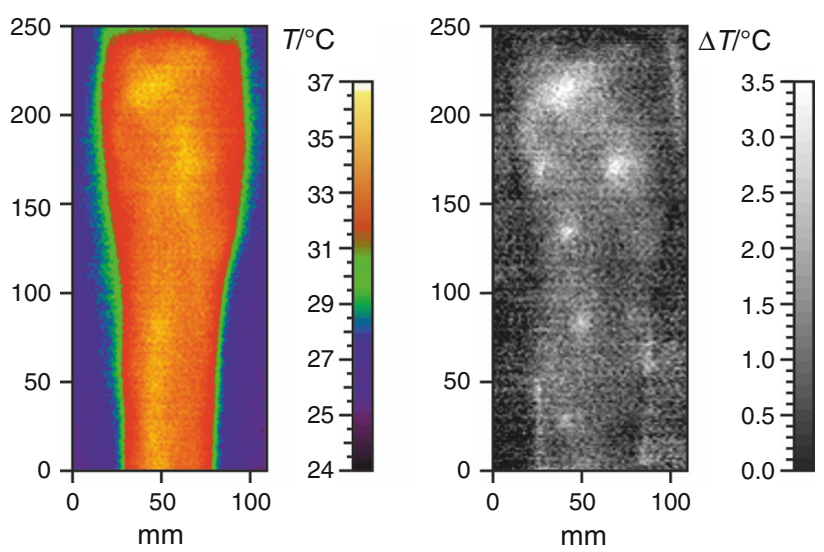

Fig. 1 Example of the infrared thermogram of the right forearm (left) as well as the result of image subtraction acquired at the end and before skin prick test (right) 
In Table 1, the average value of diagnostic TH parameter is shown for the investigated allergens. The values of diagnostic parameter are not significantly different from each other $(p=0.09)$. However, for some allergens (weeds and cat dander), average values of parameter are smaller than for others. It is due to more presence of low intensive skin responses for these allergens. The most frequently detected positive allergic responses were grasses, 7, 9 and $4 \%$ of cases for TH, SPT and sIgE methods, respectively. The most frequently detected negative results were for cat dander, dog dander and cultivated rye (6-10\%).

All categorized allergic responses were compared between each other. The gamma correlation coefficients between all considered methods are shown in Table 2.

Considering overall agreement between diagnostic methods, the best accordance was observed between $\mathrm{TH}$ and $\mathrm{sIgE}$ results (correlation coefficient $-r=0.90$ ), while TH-SPT was the most divergent pair $r=0.85$. In case of particular allergens, the biggest correlation was 0.99 , while the smallest value amounted to 0.76 .

All possible relationships between results, both positive and negative, are shown in Fig. 2 using the Venn diagram. It is clearly visible that $62 \%$ of the results of three methods overlapped. The largest number of responses non-detected by other methods was noticed for SPT (17\%), while sIgE disagreed with TH and SPT only in $0.3 \%$ of cases. Among 387 considered allergens, $1 \%$ positive reactions was detected only by the TH assessment. The percentage of the cases detected by TH and SPT methods (18\%) should also be noted. Similar relationships were obtained for particular allergens; as an example, the Venn diagram for $D$. farinae is presented (Fig. 3).

The usefulness of the TH assessment in the allergy diagnoses in respect of SPT and sIgE methods was also checked by calculations of sensitivity, specificity and accuracy. The calculations were performed for particular allergens as well as for as cases pooled together. Hence, the results for particular allergens and for cases pooled together were similar; only total results are presented in Table 3 .

It may be concluded that the TH method sensitivity is at very good level in respect of the SPT method and at good level to sIgE method. In contrast, this trend was not preserved in specificity in respect of SPT, where the value of parameter was 0.60. An acceptable level of accuracy 0.74-0.76 was also achieved in respect of both reference methods. Simultaneously, comparing SPT against sIgE method, distinctly lower values of parameters for sensitivity and accuracy, 0.56 and 0.64 , respectively, are observed than for the TH assessment.

\section{Discussion}

Our studies were focused on the validation of the $\mathrm{TH}$ method as a complementary technique in the allergy diagnosis. The validation was performed by comparison with routine diagnostic methods (SPT and sIgE). It should be noted that a perfect diagnostic procedure should have the potential to completely discriminate patients with or without disease. In case of allergy diagnosis, such method does not exist. There is even problem to distinguish a "gold standard." Hence, the TH method has been compared with two techniques commonly used in clinical practice.

The SPT and sIgE results showed good concordance to the inhalant allergens considered. Although the gamma correlations between sIgE and SPT methods (Table 2) were allergen dependent, the values ranged from 76 to $99 \%$. Previously reported data concerning comparison of SPT and sIgE [9, 11, 14] showed the agreement at the similar level of (70-97) \%. Compared to SPT and sIgE, the TH assessment is characterized by (76-96) \% accordance (Table 2). It should be emphasized that for 387 cases considered, the difference between correlation coefficients

Table 1 Diagnostic TH parameters for investigated allergens

\begin{tabular}{|c|c|c|c|c|c|}
\hline Allergen & Average & Standard deviation & Minimum & Maximum & Range \\
\hline Total & 0.79 & 0.51 & 0.11 & 2.40 & 2.28 \\
\hline Weeds & 0.63 & 0.49 & 0.12 & 2.37 & 2.25 \\
\hline Mixed trees 1 & 0.75 & 0.56 & 0.13 & 2.38 & 2.25 \\
\hline Mixed trees 2 & 0.90 & 0.55 & 0.13 & 2.40 & 2.27 \\
\hline Grasses & 0.87 & 0.51 & 0.27 & 2.23 & 1.96 \\
\hline D. pteronyssinus & 0.85 & 0.47 & 0.28 & 2.17 & 1.89 \\
\hline D. farinae & 0.91 & 0.56 & 0.23 & 2.33 & 2.10 \\
\hline Cat dander & 0.52 & 0.32 & 0.11 & 1.32 & 1.21 \\
\hline Dog dander & 0.69 & 0.43 & 0.32 & 1.99 & 1.68 \\
\hline Cultivated rye & 0.95 & 0.49 & 0.17 & 1.48 & 1.30 \\
\hline
\end{tabular}


Table 2 Gamma correlation coefficients between all investigated methods

\begin{tabular}{llll}
\hline Allergen & \multicolumn{2}{l}{ Correlation coefficient } \\
\cline { 2 - 4 } & $\begin{array}{l}\text { TH versus } \\
\text { SPT }\end{array}$ & $\begin{array}{l}\text { TH versus } \\
\text { sIgE }\end{array}$ & $\begin{array}{l}\text { SPT versus } \\
\text { SIgE }\end{array}$ \\
\hline Total & $0.85^{*}$ & $0.90^{*}$ & $0.87^{*}$ \\
Weeds & 0.91 & 0.83 & $0.76^{\times}$ \\
Mixed trees 1 & 0.87 & 0.92 & $0.79^{\times}$ \\
Mixed trees 2 & $0.81^{\times}$ & 0.92 & 0.85 \\
Grasses & 0.87 & 0.93 & 0.92 \\
D. pteronyssinus & 0.86 & 0.88 & 0.95 \\
D. farinae & 0.94 & 0.96 & 0.92 \\
Cat dander & 0.83 & 0.96 & 0.99 \\
Dog dander & 0.90 & $0.76^{\times}$ & 0.87 \\
Cultivated rye & 0.93 & 0.91 & 0.89 \\
\hline
\end{tabular}

The values marked by asterisks are statistically different. The statistical significance between particular allergens was marked by crosses. For abbreviation see text

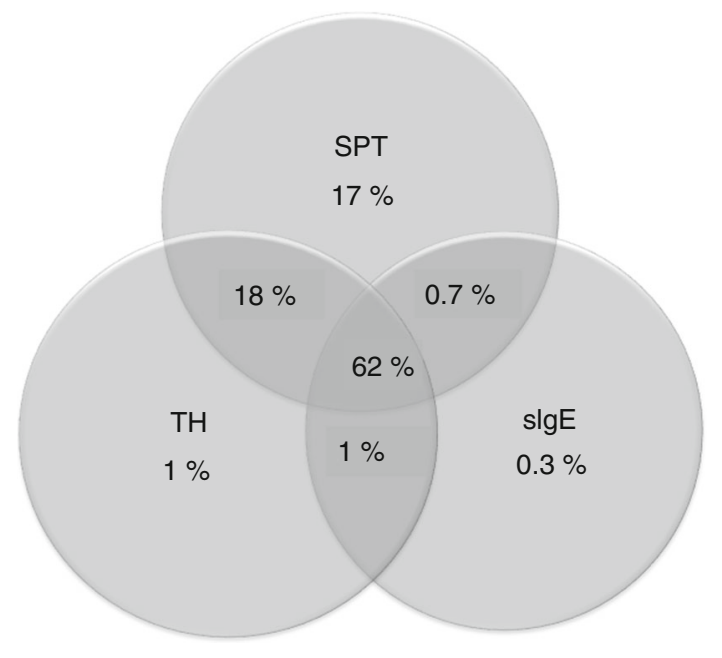

Fig. 2 Venn diagram illustrating overlapping of the results for $\mathrm{TH}$, SPT and sIgE methods. For abbreviations see text

at the level of 0.02 may be statistically significant. Therefore, the values in Table 2, marked by asterisks, are statistically different. The conclusion that SPT is better correlated with $\operatorname{SIgE}$ than with TH (Table $2-0.87$ and 0.85 , respectively) seems to be misinterpretation. In case of particular allergens, considering 43 cases, the statistical significance was found for allergen extracts marked in Table 2 by crosses.

To summarize Fig. 2, SPT method detects most cases independently (19\%). It is needed to consider why this is the case. Essentially, each considered diagnostic method detects different effects of the allergic reaction and the discrepancies between methods can be due to many factors.

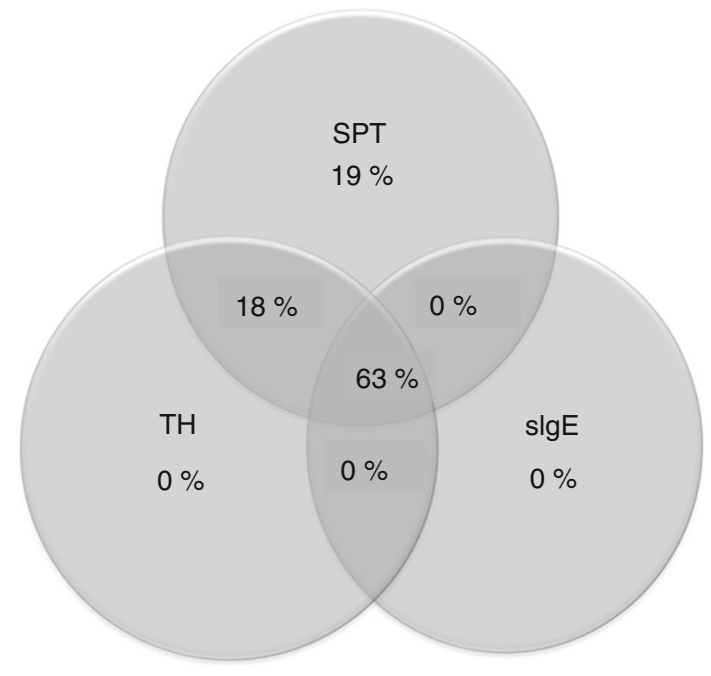

Fig. 3 Venn diagram illustrating overlapping of the results for $\mathrm{TH}$, SPT and SIgE methods for Dermatophagoides farinae

First, SPT is an in vivo method and quantifies a contact between allergen and its specific IgE antibody at the mast cell membrane, resulting in the local production of mediators and formation of wheal/flare. The difference between TH and SPT releases on the replacement of the standard quantification step (planimetry) by thermography. Additionally, SPT evaluation is mostly based on the wheal size, but its formation depends on many factors which are difficult to include in final diagnosis; as an example, the operation of lymphatic system can be given. On the other hand, $\operatorname{sgE}$ is an in vitro determination of the level of circulating IgE antibodies in serum which may be different than the skin-fixed IgE antibodies. Second, differences in the allergic quantity between the extracts used in SPT-TH and those applied for sIgE are possible. Third, different nonlinear scales are used for quantification of the allergic response.

The comparison of three methods with the use of fivepoint scales is presented in Fig. 2. Careful examination of the Venn diagram indicates the problem pertains to the small size allergic responses detected by the SPT method (about $20 \%$ positive cases). It should be kept in mind that, for small wheal diameter, the error of the diameter determination is very big $(\sim 30 \%)$ and the allergic response (value \pm error) can be assigned to two classes of the scale. The TH and SPT assessments can be classified as imaging methods from the fact that the diagnosis is based on the observation of the visual symptoms at the skin surface. In particular, in SPT method, the problematic can be also the classification of changes with extremely small size of wheal, e.g., $2 \mathrm{~mm}$ in diameter. The measurement error, in this case, rises to $50 \%$ (ruler precision). Visual assessment of such small wheal is very subjective, and the manifested 
Table 3 Sensitivity, specificity and accuracy of TH method in respect of SIgE and SPT methods and comparison between SPT and sIgE methods

\begin{tabular}{|c|c|c|c|c|c|c|c|c|c|}
\hline & \multicolumn{3}{|c|}{ Sensitivity } & \multicolumn{3}{|c|}{ Specificity } & \multicolumn{3}{|l|}{ Accuracy } \\
\hline & $\mathrm{TH} / \mathrm{sIgE}$ & TH/SPT & SPT/sIgE & $\mathrm{TH} / \mathrm{sIgE}$ & TH/SPT & SPT/sIgE & $\mathrm{TH} / \mathrm{sIgE}$ & TH/SPT & $\mathrm{SPT} / \mathrm{sIgE}$ \\
\hline Total & 0.72 & 0.93 & 0.56 & 0.88 & 0.60 & 0.93 & 0.76 & 0.74 & 0.64 \\
\hline
\end{tabular}

For abbreviations see text

change can be the result of an allergic reaction as well as the response of the skin to prick. This may result in misinterpretation of allergic responses, which routinely are classified as 1 in five-point scale, while other methods classified them as negative. Obviously, it does not make any difference from clinical point of view but causes discrepancies between considered methods. Such overestimation concerned about $9 \%$ of considered allergic cases for SPT evaluation. Generally, in case of SPT, sometimes regarded as a "gold standard" [10], the risk of making a mistake is significantly higher than for $\mathrm{TH}$, which is a digital technique. The human factor and experience of medical staff can significantly affect the SPT results. The remaining discrepancies are characterized by the 2-level differences of the allergic responses and cannot be correlated with the experimental errors. The above rigorous analysis is not still satisfying because it does not allow to full explanation of about $10 \%$ misinterpreted cases by SPT method.

At this point very useful was the model analysis of thermograms which provides opportunity to determine the migration rate of the basic mediator of allergic reactionhistamine $(v)[12,13]$. Temporal acquisition of control histamine response allows estimating the migration rate of histamine $(v)$ in the skin (average $v=0.013 \pm 0.010 \mathrm{~mm} /$ $\mathrm{s}$, range: $(0.007-0.041) \mathrm{mm} / \mathrm{s})$. The inter-subject variability of the parameter can be considered as a confirmation that the immediate allergic response is a highly individual-dependent process, but its value can reflect the skin reactivity. Previous studies have confirmed that histamine migration rate is also parameter responsible for the maximal radius of the heated region (correlation coefficient $=0.85)[12,13]$. A comparison of the histamine migration rate with age of patients seems to be interesting (Fig. 4). It was reported that in some cases allergy diagnosis, based on the SPT, can be difficult due to low or high skin reactivity. For example, in the elderly patients, a problem of correct diagnosis is the subject of recent studies [15]. Many authors claim that skin reactivity to allergens decreases with age [16].

Routine SPT does not consider the skin reactivity, while the TH assessment offers such possibility. The migration rate of control histamine may be treated as a measure of skin reactivity and may be extracted from the analysis of the thermographic data. As it is shown in Fig. 4, the

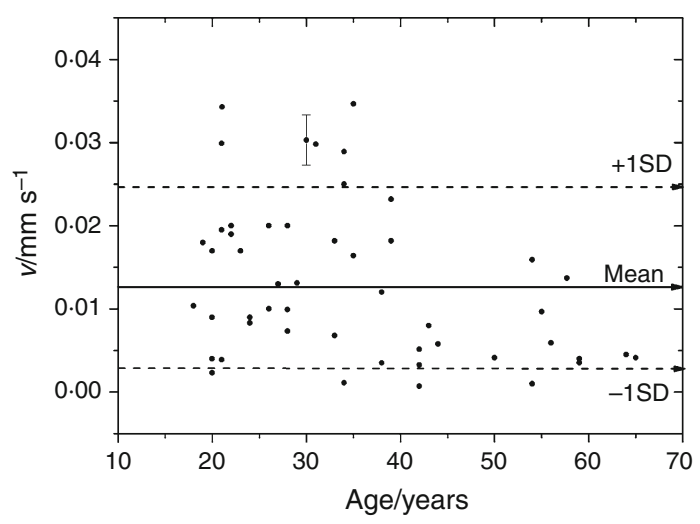

Fig. 4 Dependence between migration rate of histamine and age of patients. Error bars mark the experimental errors

histamine migration rate is a highly specific parameter which may affect final diagnosis. Considering its values, both hypersensitive patients $(v>$ mean $+\mathrm{SD})$ can be considered as well as those with low skin reactivity $(v<$ mean - SD). In Fig. 4, many cases larger than mean + SD can be distinguished, while only two cases with extremely low skin reactivity were observed. Since in the SPT evaluation the fundamental parameter, describing allergic response, is the diameter of the wheal, this parameter was compared between hypersensitivity $(v>$ mean $+\mathrm{SD}) \quad$ and "normal" patients $(v<$ mean $+\mathrm{SD})$ and statistically significant differences were observed $(p=0.0018)$. It may be interpreted that for hypersensitive patients, large sizes of skin reactions could result from the high skin reactivity than from the allergic reaction itself. For these patients, allergic responses determined by SPT were one level bigger than detected by the TH assessment. Such overestimation concerned $5 \%$ of considered allergic cases (Fig. 2). The TH quantification automatically considers migration rate of histamine in the final outcome of diagnosis, while in SPT evaluation, the impact of the skin condition on test results cannot be eliminated. Unfortunately, low statistics (small positive allergic cases) does not allow calculations for patients with low skin reactivity. Additionally, considering the relationship between the skin reactivity and age, it cannot be distinguish an explicit trend (Fig. 4); however, all hypersensitivity cases can be found in the age group up to 40 years ( 7 cases). 
It is clear that application of the above corrections reduces the number of misinterpreted cases by SPT. Although the allergic responses are commonly quantified in a $0-4$ scale, clinically useful cases are those that are smaller or larger than a certain fixed level (e.g., in SPT method-smaller or larger wheal diameter than control). To demonstrate the influence of the scale selection of the results and consideration of proposed corrections, the Venn diagram was recalculated using a new three-point scale. Following limits were adopted: $0-$ negative response, $1-$ positive (untreated) response equivalent to 1 and 2 on the categorized scale and 2-positive (treated) response equivalent to 3 and 4 on the categorized scale. Applying a new three-point scale for allergic responses, the Venn diagram has been calculated (Fig. 5).

Comparison of Fig. 2 with Fig. 5 clearly shows, that, from clinical point of view, application of five-point scale evaluation can be successfully substituted by three-point scale which is sufficient. The changes of the applied scales (Fig. 5) and introduction of corrections increase the number of overlapped cases to $89 \%$ and significantly reduce allergic responses detected only by SPT and SPT-TH methods.

Applying this approach and comparing the overall relationships of positive allergic cases to negative cases, for particular methods, with their equivalents for the individual allergens, almost identical relationships, in the range of error, were observed. As an example, a cultivated rye can be given, where average ratio of positive cases to negative cases is at the level of $0.16 \pm 0.05$ for $\mathrm{TH}$ assessment, while global value of this ratio was noted at the level of 0.15 . It can be concluded that particular methods are allergen independent.

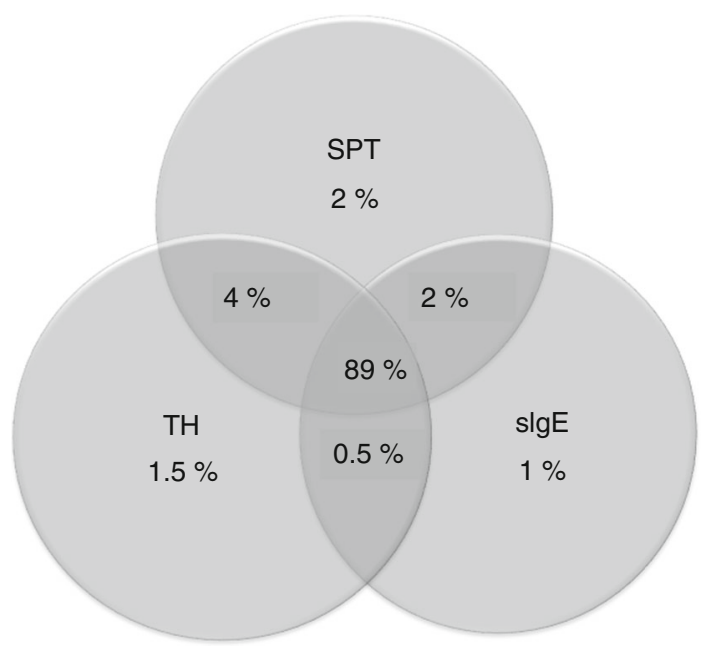

Fig. 5 Venn diagram illustrating overlapping the results of SPT, TH and sIgE methods. The diagram was constructed using a three-point scale. For abbreviations see text
To compare the TH assessment with SPT and sIgE tests, the standard statistical parameters sensitivity, specificity and accuracy were calculated (Table 3). As a complement, these parameters were calculated for both reference methods. In the interpretation of the results presented in Table 3, the differences in positive and negative cases detected by SPT and SIgE methods should be kept in mind (Fig. 2). For most allergens tested as well as in case of all allergens pooled, sensitivity of the TH assessment compared to SIgE (0.72) and SPT (0.93) is at good level. With regard to specificity, TH is not so effective, especially in respect of the SPT evaluation (0.60). It should be noted that in the calculation of specificity only patients without the disease are considered. Due to possible overestimation by the SPT method, the number of false positives cases assessed by the TH method increases. It is probably the reason for lower value of specificity. The calculations of accuracy yield 0.76 and 0.74 results for sIgE and SPT, respectively. The results of accuracy should be, however, handled with care. In this case, population with a low disease prevalence was studied and this only means that in absolute number the TH method gives correct classified results. Full verification of accuracy would require studies for a larger group of allergic patients with positive responses. It should be also noted that comparison of these statistical parameters between both reference methods gives significantly lower values of sensitivity and accuracy, at the level of $0.56-0.64$ in total. The exception was observed for specificity, but not for all allergens, remaining at the comparable 0.93 level on average.

Additionally, the great advantage of TH method compared to routine applied methods is the size of experimental error. Due to high sensitivity of infrared cameras, size of the heated areas is usually larger than size of lesions identified visually. TH experimental error is determined by pixel size of the thermogram. Therefore, the precision of lesion measurement on thermogram is $5-7 \%$. While in the routinely methods, the errors are not determined. It can be notably significant, especially in the SPT, where the planimetric measurement of the wheal diameter is limited by the precision of the ruler, and it is strongly limited by the technician experience. Allergen-specific immunoglobulin $\mathrm{E}$ detection and quantification have become an important step in allergy detection and is validated according to the accreditation procedure under the EN ISO 15189 standard [2]. Generally, the results are given without errors, but recently the studies for method repeatability at different laboratories were performed. The mean coefficient of variation for all allergens, all sIgE concentrations and all laboratories was at the level of $10 \%$ [2].

The collected results indicate unequivocally that thermographic assessment supplemented by a mathematical model may be treated as the adjuvant tool in the diagnosis 
of allergy. The method can being accused that is only an additional and SPT-dependent test. Additional advantages of the TH assessment are the consideration of patient's skin condition and calculations of the experimental errors.

Nevertheless, the application of thermographic method during SPT is a complementary tool to diagnose allergy. The stabilization of patient's skin temperature, before examination, slightly extends the time of testing. Additionally, an essential software and thermocamera are needed. Also, an additional time for analysis and interpretation of results should be considered.

In summary, verification of the $\mathrm{TH}$ assessment against commonly used methods in allergy diagnosis gives promising results. Excellent agreement between SPT and sIgE methods was observed and makes the TH assessment competitive. Moreover, the errors of results are well defined and precision of diagnosis is kept at constant level. Additional advantage of the TH method relies on an estimation of the skin reactivity which allows to emphasis the hypersensitivity patients and automatic correction of the diagnosis. Relatively low costs of infrared camera enhance its availability, and nowadays, it seems to be natural consequence of digital technology development. In recent years, applications of thermography as a noninvasive method have become more popular and can be even observed in different areas of sport medicine and science [17-19].

Acknowledgements This research was supported by Grant K/DSC/ 000715 .

Open Access This article is distributed under the terms of the Creative Commons Attribution 4.0 International License (http://crea tivecommons.org/licenses/by/4.0/), which permits unrestricted use, distribution, and reproduction in any medium, provided you give appropriate credit to the original author(s) and the source, provide a link to the Creative Commons license, and indicate if changes were made.

\section{References}

1. Heinzerling L, Mari A, Bergmann KC, Bresciani M, Burbach G, Darsow U, Durham S, Fokkens W, Gjomarkaj M, Haahtela T, Bom AT, Wohrl S, Maibach H, Lockey R. The skin prick testEuropean standards. Clin Transl Allergy. 2013;3(3):7022-3-3.

2. Lambert C, Sarrat A, Bienvenu F, Brabant S, Nicaise-Roland P, Alyanakian MA, Apoil PA, Capron C, Couderc R, Evrard B, Jaby D, Hemont C, Laine C, Lelong M, Mariotte D, Martinet J, Renier G, Sainte-Laudy J, Tabary T, Treiner E, Uring-Lambert B, Vigneron C, Vivinus M, Witthuhn F, Vitte J. AllergoBioNet sIgE accreditation interest group. The importance of EN ISO 15189 accreditation of allergen-specific IgE determination for reliable in vitro allergy diagnosis. Allergy. 2015;70:180-6.
3. de Vos G, Nazari R, Ferastraoaru D, Parikh P, Geliebter R, Pichardo Y, Wiznia A, Rosenstreich D. Discordance between aeroallergen specific serum IgE and skin testing in children younger than 4 years. Ann Allergy Asthma Immunol. 2013;110:438-43.

4. de Vos G. Skin testing versus serum-specific IgE testing: which is better for diagnosing aeroallergen sensitization and predicting clinical allergy? Curr Allergy Asthma Rep. 2014;14:430-8.

5. De Vera MJ, Drapkin S, Moy JN. Association of recurrent wheezing with sensitivity to cockroach allergen in inner-city children. Ann Allergy Asthma Immunol. 2003;91:455-9.

6. Rabito FA, Carlson J, Holt EW, Iqbal S, James MA. Cockroach exposure independent of sensitization status and association with hospitalizations for asthma in inner-city children. Ann Allergy Asthma Immunol. 2011;106:103-9.

7. Schoos AM, Chawes BL, Folsgaard NV, Samandari N, Bonnelykke K, Bisgaard H. Disagreement between skin prick test and specific IgE in young children. Allergy. 2015;70:41-8.

8. de Vos G, Nazari R, Ferastraoaru D, Parikh P, Geliebter R, Pichardo Y, Wiznia A, Rosenstreich D. Discordance between aeroallergen specific serum $\operatorname{IgE}$ and skin testing in children younger than 4 years. Ann Allergy Asthma Immunol. 2013;110:438-43.

9. Cho JH, Suh JD, Kim JK, Hong SC, Park IH, Lee HM. Correlation between skin-prick testing, individual specific IgE tests, and a multiallergen IgE assay for allergy detection in patients with chronic rhinitis. Am J Rhinol Allergy. 2014;28:388-91.

10. Asha'ari ZA, Suhaimi Y, Yusof RA, Rushdan I, Maraina $\mathrm{CH}$. Comparison of serum specific IgE with skin prick test in the diagnosis of allergy in Malaysia. Med J Malaysia. 2011;66: 202-6.

11. Calabria CW, Dietrich J, Hagan L. Comparison of serum-specific IgE (ImmunoCAP) and skin-prick test results for 53 inhalant allergens in patients with chronic rhinitis. Allergy Asthma Proc. 2009;30:386-96.

12. Rokita E, Rok T, Taton G. Application of thermography for the assessment of allergen-induced skin reactions. Med Phys. 2011;38:765-72.

13. Rokita E, Rok T, Tatoń G. Evaluation of allergic response using dynamic thermography. Opto-Electron Rev. 2015;1:53-9.

14. Gupta NKR. Skin prick test versus specific IgE-a comparative study in patients with bronchial asthma and allergic rhinitis in India. Eur Respir J. 2014;44(Suppl):P4033.

15. Scichilone N, Callari A, Augugliaro G, Marchese M, Togias A, Bellia V. The impact of age on prevalence of positive skin prick tests and specific IgE tests. Respir Med. 2011;105:651-8.

16. King MJ, Lockey RF. Allergen prick-puncture skin testing in the elderly. Drugs Aging. 2003;20:1011-7.

17. Boerner E, Bauer J, Ratajczak B, Dereń E, Podbielska H. Application of thermovision for analysis of superficial temperature distribution changes after physiotherapy. J Therm Anal Calorim. 2015;120:231-67.

18. Cholewka A, Kasprzyk T, Stanek A, Sieron-Stołtny K, Drzazga Z. May thermal imaging be useful in cyclist endurance test? J Therm Anal Calorim. 2016;123:1973-9.

19. Ratajczak B, Boerner E. Application of thermovision in assessment of superficial tissue temperature changes under the influence of $1 \mathrm{MHz}$ and $3 \mathrm{MHz}$ ultrasounds wave. J Therm Anal Calorim. 2015;120:269-75. 